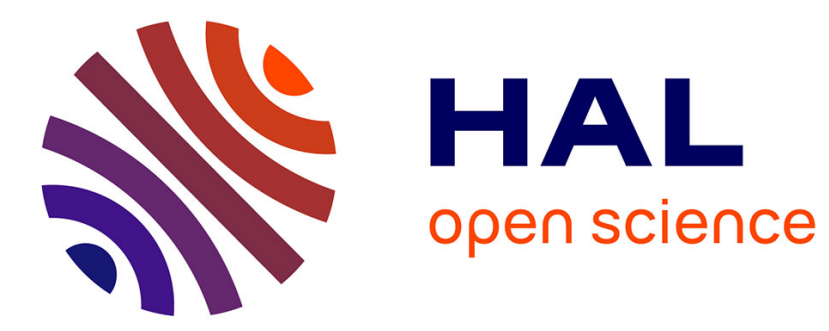

\title{
From light-scattering measurements to polarizability derivatives in vibrational Raman spectroscopy: The 2 nu(5) overtone of SF6
}

David Kremer, Florent Rachet, Michel Chrysos

\section{- To cite this version:}

David Kremer, Florent Rachet, Michel Chrysos. From light-scattering measurements to polarizability derivatives in vibrational Raman spectroscopy: The $2 \mathrm{nu}(5)$ overtone of SF6. Journal of Chemical Physics, 2013, 138 (17), Non spécifié. 10.1063/1.4803160 . hal-03344721

HAL Id: hal-03344721

https://univ-angers.hal.science/hal-03344721

Submitted on 15 Sep 2021

HAL is a multi-disciplinary open access archive for the deposit and dissemination of scientific research documents, whether they are published or not. The documents may come from teaching and research institutions in France or abroad, or from public or private research centers.
L'archive ouverte pluridisciplinaire HAL, est destinée au dépôt et à la diffusion de documents scientifiques de niveau recherche, publiés ou non, émanant des établissements d'enseignement et de recherche français ou étrangers, des laboratoires publics ou privés. 


\section{AD| $\begin{aligned} & \text { The Journal of } \\ & \text { Chemical Physics }\end{aligned}$}

\section{From light-scattering measurements to polarizability derivatives in vibrational Raman spectroscopy: The 2v5 overtone of SF6}

D. Kremer, F. Rachet, and M. Chrysos

Citation: The Journal of Chemical Physics 138, 174308 (2013); doi: 10.1063/1.4803160

View online: http://dx.doi.org/10.1063/1.4803160

View Table of Contents: http://scitation.aip.org/content/aip/journal/jcp/138/17?ver=pdfcov

Published by the AIP Publishing

\section{Articles you may be interested in}

More light on the 2v5 Raman overtone of SF6: Can a weak anisotropic spectrum be due to a strong transition anisotropy?

J. Chem. Phys. 140, 034308 (2014); 10.1063/1.4861047

Field-resolved measurement of reaction-induced spectral densities by polarizability response spectroscopy

J. Chem. Phys. 127, 184505 (2007); 10.1063/1.2792943

Isotropic and anisotropic collision-induced light scattering by gaseous sulfur hexafluoride at the frequency region of the $v 1$ vibrational Raman line

J. Chem. Phys. 118, 11009 (2003); 10.1063/1.1575733

The 5th- and 7th-order 2D Raman spectroscopy for intramolecular vibrational modes

AIP Conf. Proc. 503, 144 (2000); 10.1063/1.1302858

Coherent two-dimensional Raman scattering: Frequency-domain measurement of the intra- and intermolecular vibrational interactions

J. Chem. Phys. 108, 1326 (1998); 10.1063/1.475505

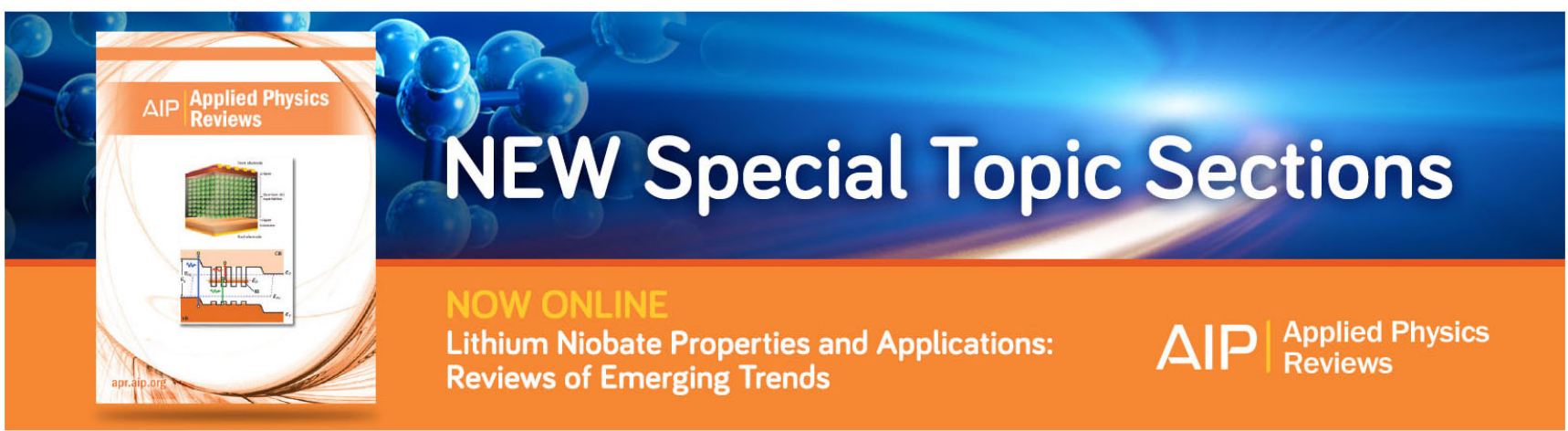




\title{
From light-scattering measurements to polarizability derivatives in vibrational Raman spectroscopy: The $2 v_{5}$ overtone of $\mathrm{SF}_{6}$
}

\author{
D. Kremer, F. Rachet, and M. Chrysos a) \\ LUNAM Université, Université d'Angers, CNRS UMR 6200, Laboratoire MOLTECH-Anjou, \\ 2 Bd Lavoisier, 49045 Angers, France
}

(Received 23 March 2013; accepted 16 April 2013; published online 7 May 2013)

\begin{abstract}
The room-temperature isotropic spectrum of $\mathrm{SF}_{6}$ was recorded at the frequency of the $2 v_{5}$ overtone by running high-sensitivity incoherent Raman experiments for two independent polarizations of the incident beam and for gas densities varying from 2 to 27 amagat. Weak yet observable pressure effects were found. A transparent analysis of the Raman cross-section problem along with the first-ever prediction of the value of the mean polarizability second derivative $\partial^{2} \bar{\alpha} / \partial q_{5}^{2}$ are made and the hitherto underestimated role of the hot bands of $\mathrm{SF}_{6}$ is brought to the wider public. The emergence of an analytic hotband factor is shown whose magnitude is dramatically increased with the order of the overtone and the gas temperature and all the more so upon considering low-frequency molecular vibrations. Our formulas, which in the harmonic approximation are exact, are still applicable to real situations provided certain conditions are fulfilled. For nondegenerated modes, generalization to higher order overtones is made, an issue addressing the much challenging problem of the IR-allowed second overtone bands. The content of this paper is also an invitation towards ab initio derivative-calculations for sulfur hexafluoride, especially given the today's needs in interpreting spectra of significance for greenhouse atmospheric issues. (C) 2013 AIP Publishing LLC. [http://dx.doi.org/10.1063/1.4803160]
\end{abstract}

\section{INTRODUCTION}

Vibrational Raman and IR spectroscopy in nonpolar compressed molecular gases, though among the age-old techniques, still proves the best way to access dipole polarizability and dipole moment derivatives with respect to the vibration normal coordinates of a molecule ${ }^{1-3}$ (see also Refs. 4-8, and references therein). Within that spectroscopy, frequencyresolved spectra, generated by the oscillating dipole-dipole polarizability $\alpha$ or dipole moment $\mu$, are recorded around the frequency of a vibrational transition, from which a lot can be learnt about the way in which the molecule oscillates at that frequency. It happens often that the absorption or scattering cross-section of a vibrational transition is too small a value to emerge from the noise, thus requiring high gas pressures to make enough intense signals appear. In doing so, however, line spectra start to overlap due to neighboring transitions, resulting in a spectrum that looks more like a broad bump than like anything described as a sharp spectral line at the expected frequency. ${ }^{9}$ Serious difficulties may thus appear with the interpretation. This is even more so when the equipment used to record the intensities is not designed for high resolution imaging, and spectral resolutions of the order of $\sim 1 \mathrm{~cm}^{-1}$ are only possible. Although such values are too low a performance in comparison to some of today's feats of instrumentation, ${ }^{10-12}$ it is sometimes the cost of the effort required to maximize sensitivity, as in the case of the very high-sensitivity Raman equipment of our institute. ${ }^{13-17}$

To make things concrete, let us take $\mathrm{SF}_{6}$ and its $2 v_{5}$ Raman overtone. This molecule has been witnessing re-

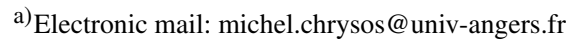

newed spectroscopic interest in the last two decades, ${ }^{18-25}$ with its role in environmental and atmospheric issues being among the reasons for concern. ${ }^{26-28}$ At room temperature, the overtone $2 v_{5}$ manifests itself as a highly polarized weak-intensity spectrum, ${ }^{29,30}$ which is almost completely drowned out in a plethora of hot or difference band transitions that all are simultaneously "excited" at nearly the same frequency $\left(\sim 1048 \mathrm{~cm}^{-1}\right)$. Whereas in all those transitions the vibrational quantum number associated with $\nu_{5}$ makes a jump from $v_{5}=0$ to $v_{5}=2$, one or several $v_{i}$ $(i \neq 5)$ modes of the molecule may have remained excited in an arbitrary vibrational level $v_{i}$ during the transition. Matrix elements such as $\langle 000021|\alpha| 000001\rangle,\langle 000022|\alpha| 000002\rangle$, $\langle 000120|\alpha| 000100\rangle$ are typical of such a type of bands, taken arbitrarily from the pool of transitions around the frequency value $1048 \mathrm{~cm}^{-1}$. These are identified as the hot bands $v_{6}$ $+2 v_{5}-v_{6}, 2 v_{6}+2 v_{5}-2 v_{6}$, and $v_{4}+2 v_{5}-v_{4}$, respectively, and in a way analogous to many others, they can greatly affect the spectrum of the "pure $2 v_{5}$ overtone" and wrongly be thought of as pertaining to the transition $\langle 000020|\alpha| 000000\rangle$. Worse, a multitude of other hot bands also occurs at nearly the same frequency, owing to an initially excited vibrational level $v_{5}$. Among the large number of such bands, one may quote the $3 v_{5}-v_{5}$, the $4 v_{5}-2 v_{5}$, or even combinations of the type $v_{6}+3 v_{5}-\left(v_{6}+v_{5}\right)$ or $2 v_{6}+3 v_{5}-\left(2 v_{6}+v_{5}\right)$, with matrix elements $\langle 000030|\alpha| 000010\rangle,\langle 000040|\alpha| 000020\rangle$, $\langle 000031|\alpha| 000011\rangle$, and $\langle 000032|\alpha| 000012\rangle$, respectively. The question then arises: Is it possible to isolate from the experimentally measured integrated cross-section the bare overtone contribution $\langle 000020|\alpha| 000000\rangle$ ? In many systems, there is a reduced number of hot bands in the vicinity of a transition, so what in fact one measures, there, is to a good 
approximation the real transition alone. In those cases there is not even a question to answer. But in the case of $\mathrm{SF}_{6}$ (especially for low-frequency bending modes) things can be very different. This is even more so upon treating hot gas. Not only the severe coalescence that occurs at high gas pressure or temperature conditions makes it impossible to isolate $\langle 000020|\alpha| 000000\rangle$, but even if it were possible to do so it would be of limited interest, resulting in matrix elements that are temperature-dependent. What in fact matters in making such an experiment is to extract information inherent in the molecule, namely, some value for the physically relevant effective second polarizability derivative $\left(\partial^{2} \alpha / \partial q_{i}^{2}\right)_{\text {eff }}$ (Ref. 31). This derivative is, by construction, the same for all the matrix elements pertaining to the spectrum, which will only differ in proportionality factors that can be worked out analytically.

It is the purpose of this paper to report on the link between the integrated overtone spectrum $M_{0}$ and the harmonic property $\left(\partial^{2} \alpha / \partial q_{i}^{2}\right)$. In the harmonic approximation these formulas are exact. In the presence of anharmonicity, $\left(\partial^{2} \alpha / \partial q_{i}^{2}\right)_{\text {eff }}$ should instead be viewed as the anharmonic derivative in the $\langle 000020|\alpha| 000000\rangle$ transition, which differs from $\left(\partial^{2} \alpha / \partial q_{i}^{2}\right)$ in ( $v$-independent) anharmonic corrections. In certain situations, such as for modes $v_{i}$ and conditions ensuring $\frac{h v_{i}}{k_{B} T} \ll 1$, weakly coupled vibrations and small cubic force constants $k_{k i i}(k \neq i)$, as in the case treated in the example to be developed below, the harmonic approximation is enough. Yet, even in the most unfavorable cases, the content of this paper will be of value for assessing the otherwise unsuspected degree of difficulty in treating a multiply degenerated low-energy bending mode of a high-symmetry molecule, which by nature is closer to an infinitely degenerated gas than to an ordinary few-levels textbook system.

\section{THEORY}

\section{A. Past and current trends}

There is long evidence that absorption or scattering crosssections can increase with temperature. Reports have appeared in the 1960s claiming an increase by a factor 1000 in the mean polarizability of molecular hydrogen upon heating the gas to $5000 \mathrm{~K}$ and averaging the vibrational ${ }^{32}$ and rotational ${ }^{33,34}$ states. Surprising effects such as the "cold-band effect" were seen experimentally, in the early 1980s, in which the $3 v_{3}$ absorption cross-section of $\mathrm{SF}_{6}$ was found to decrease with increasing temperature. ${ }^{35} \mathrm{~A}$ few years later, absorption experiments with $\mathrm{SF}_{6}$ showed the existence of enhanced hotband multiphoton absorption. ${ }^{36}$ The use of cryostatic cells and jet-cooled equipment to reduce the effect of hot bands also goes back to that time $\mathrm{e}^{37,38}$ and still continues nowadays..$^{39,40}$ Late in the 1980s, refraction index experiments, carried out by Hohm and Kerl, have shown how to monitor the temperature dependence of electronic polarizability for molecular and atomic gases. ${ }^{41,42}$ As for theory, internal-coordinate formulations for the vibration-rotation energies of octahedral spherical top molecules have allowed to determine partial sets of cubic potential energy coefficients in the normal and symmetry coordinate bases. ${ }^{43}$ Similar sets of data were almost simultaneously reported by other scientists, on the basis of experiments or calculations for $\mathrm{SF}_{6}$ (Refs. 44 and 45). More recently, new techniques for constructing representations of point groups, that are particularly useful when describing high overtones, have been introduced. ${ }^{46}$

In what follows, we focus on sulfur hexafluoride and on its vibrational spectra and derivatives, which are timely issues to deal with. ${ }^{27,28}$ Of the six vibration modes of $\mathrm{SF}_{6}$, only $v_{3}$ and $v_{4}$ are IR active, and thus directly involved in radiative forcing. Pure bending modes, such as the scissoring vibration $v_{5}$ on which our paper mainly focuses, do not directly involve deformation of the S-F bond. This fact explains the origin of the believed small or even tiny value of the anharmonicity constant $k_{155}\left[=\frac{\phi_{155}}{2}=C_{155}=-17 \mathrm{~cm}^{-1}\right.$ (Ref. 44), $0.68 \mathrm{~cm}^{-1}$ (Ref. 45)] and of the difficulty in obtaining reliable data for that quantity. ${ }^{47}$ In this respect, Krohn and Overend have come to the conclusion that their "calculated values of $C_{155}$ and $C_{166}$ may not be reliable" and that "incompleteness in the bending and stretch-bend anharmonicities is responsible for shortcomings in calculations involving the lower-frequency modes." ${ }^{44}$ Similar are the conclusions of Hodgkinson, Barrett, and Robiette, ${ }^{45}$ who have pointed out that cubic constants which only involve the interactions of stretching vibrations are an order of magnitude larger than those involving the bending vibration $v_{5}$, and that their "general model is not particularly accurate for stretch-bend constants."Although the need for improved spectroscopic data and for explicit account of stretch-bend and bend-bend interactions in the force field of $\mathrm{SF}_{6}$ appears clearly already in the mid-1980s (Ref. 45, p. 945), 25 years later there is still no improvement in this area. This need is timelier today than ever before in view of the recent conclusions of NASA scientists: the bond derivatives (especially the ones involving fluorine atoms) have been identified as being responsible for the molecular origin of greenhouse warming. ${ }^{48}$

\section{B. Vibrational partition functions and populations}

To represent the states over which the vibrational energy, $E_{\vec{v}}$, of the gas molecules is distributed in thermodynamic equilibrium, $m$-uplets $\vec{v}=\left(v_{1}, v_{2}, \ldots, v_{m}\right)$ pertaining to the ensemble $\mathbb{N}^{m}$ are used, where $m$ is the number of molecular normal modes and $v_{i}$ are the associated vibrational quantum numbers. For $\mathrm{SF}_{6}, m=6$. According to Boltzmann statistics, the fraction of $\mathrm{SF}_{6}$ molecules occupying a sixuplet $\vec{v}$, at temperature $T$, reads:

$$
P_{\vec{v}}=\frac{1}{Z} \prod_{i=1}^{m} g_{n_{i}, i}\left(v_{i}\right) e^{-v_{i} x_{i}},
$$

where $x_{i}=\frac{h c v_{i}}{k_{B} T}$ and

$$
Z=\sum_{v_{i}=0}^{\infty} \prod_{i=1}^{m} g_{n_{i}, i}\left(v_{i}\right) e^{-v_{i} x_{i}}
$$

is the total vibrational partition function in which the contribution of the zero-point vibrational energy has been removed (more about this intentional omission is said below). In the above formulas, $v_{i}$ is the fundamental frequency of mode $i$, 
and $n_{i}$ its degeneracy. $g_{n_{i}, i}\left(v_{i}\right)$ stands for the degeneracy of the $v_{i}$ th excited state of this mode. The calculation of $g_{n_{i}, i}\left(v_{i}\right)$, for a molecule vibrating harmonically in an $n_{i}$-fold degenerated mode $v_{i}$, addresses notions of combinatorics to answer the question: "in how many different ways can one satisfy $a_{1}+a_{2}+\ldots+a_{n_{i}}=v_{i}$, where $a_{1}, a_{2}, \ldots a_{n_{i}}$ are nonnegative integers?" In the case of $i=1$, which, in both $\mathrm{SF}_{6}$ and $\mathrm{CO}_{2}$, is a totally symmetric vibration, $v_{1}$ is a nondegenerated mode $\left(n_{1}=1\right)$ and there is only one way to satisfy the aforementioned condition. As a result, $g_{n_{1}, 1}\left(v_{1}\right)=1$ whatever the value $v_{1}$. In the case of $v_{2}(i=2)$, which for both those molecules is a doubly degenerated mode $\left(n_{2}=2\right)$, there are obviously $g_{n_{2}, 2}\left(v_{2}\right)=v_{2}+1$ ways to satisfy the condition $a_{1}+a_{2}=v_{2}$. Finally, in the case of the vibrations $v_{3}, v_{4}, v_{5}$, and $v_{6}$ of $\operatorname{SF}_{6}(i=3, \ldots, 6)$, which are all triply degenerated $\left(n_{i}=3\right)$, one can readily check that there are $g_{n_{i}, i}(v)=\frac{1}{2}\left(v_{i}+1\right)\left(v_{i}+2\right)$ ways to satisfy $a_{1}+a_{2}+a_{3}=v_{i}$. The three specific formulas given above for $n_{i}=1,2$, and 3 can readily be generalized to any value of $n_{i}$ through the formula: ${ }^{49}$

$$
g_{n_{i}, i}\left(v_{i}\right)=\frac{\left(v_{i}+n_{i}-1\right) !}{\left(n_{i}-1\right) ! v_{i} !}
$$

Table I gathers, in the order of decreasing probability, the 33 sixuplets $\vec{v}$ that were necessary for convergence to $95 \%$ of the fractional population of $\mathrm{SF}_{6}$ at room temperature, along with degeneracy $g(\vec{v})\left[=\prod_{i=1}^{6} g_{n_{i}, i}\left(v_{i}\right)\right]$, vibrational energy $E_{\vec{v}}$ (in $\mathrm{cm}^{-1}$ ), and occupation probability $P_{\vec{v}}$ for the state $\vec{v}$ at that temperature. At $295 \mathrm{~K}$, only $32 \%$ of the molecules are in the ground vibrational state. The next three excited states, (000001), (000010), and (000002), contain together as many molecules as the ground state itself. The missing $5 \%$ of the population is spread over a huge number of states (not shown). More than $15 \%$ of the total population is distributed over levels that are only very little occupied $(<1 \%)$. Similar data, but limited to the 15 most populated states, have been reported in the past; ${ }^{50}$ wherever the comparison is possible, excellent agreement between the two series of data is found.

Rearrangement between the product and the sum operators in the expression of Eq. (2) allows one, with the help of Eq. (3), to deduce the very useful formula: ${ }^{51}$

$$
Z=\prod_{i=1}^{m}\left(Z_{i}\right)^{n_{i}}
$$

where $Z_{i}=\left(1-e^{-x_{i}}\right)^{-1}$ is the vibrational partition function for a single degree of freedom of vibration $i$. As mentioned above, the zero-point vibrational energy is missing in the expression $Z_{i}=\left(1-e^{-x_{i}}\right)^{-1}$, which should instead read: $Z_{i}=e^{-\frac{x_{i}}{2}}\left(1-e^{-x_{i}}\right)^{-1}$. Yet there are good reasons why we might want to remove this factor from the definition of $Z_{i}$ (Ref. 52).

Application of Eq. (4) to the case of $\mathrm{SF}_{6}$ results in the very useful and simple expression:

$$
\begin{aligned}
Z= & \left(1-e^{-x_{1}}\right)^{-1}\left(1-e^{-x_{2}}\right)^{-2} \\
& \times\left[\left(1-e^{-x_{3}}\right)\left(1-e^{-x_{4}}\right)\left(1-e^{-x_{5}}\right)\left(1-e^{-x_{6}}\right)\right]^{-3}
\end{aligned}
$$

TABLE I. States of $\mathrm{SF}_{6}$ sharing, at $295 \mathrm{~K}, 95 \%$ of the total gas population.

\begin{tabular}{|c|c|c|c|}
\hline$\vec{v}$ & $g(\vec{v})$ & $E\left(\mathrm{~cm}^{-1}\right)$ & $P_{\vec{v}}(\%)$ \\
\hline$(000000)$ & 1 & 0 & 31.839 \\
\hline$(000001)$ & 3 & 348.08 & 17.490 \\
\hline (000010) & 3 & 523.56 & 7.432 \\
\hline (000002) & 6 & 696.16 & 6.405 \\
\hline (000100) & 3 & 615.02 & 4.758 \\
\hline$(000011)$ & 9 & 871.64 & 4.083 \\
\hline (010000) & 2 & 643.35 & 2.762 \\
\hline (000101) & 9 & 963.10 & 2.614 \\
\hline$(000003)$ & 10 & 1044.24 & 1.955 \\
\hline (010001) & 6 & 991.43 & 1.518 \\
\hline$(000012)$ & 18 & 1219.72 & 1.495 \\
\hline$(000020)$ & 6 & 1047.12 & 1.157 \\
\hline (000110) & 9 & 1138.58 & 1.111 \\
\hline (000102) & 18 & 1311.18 & 0.957 \\
\hline (001000) & 3 & 947.98 & 0.938 \\
\hline$(100000)$ & 1 & 774.55 & 0.728 \\
\hline (010010) & 6 & 1166.91 & 0.645 \\
\hline$(000021)$ & 18 & 1395.20 & 0.635 \\
\hline (000111) & 27 & 1486.66 & 0.610 \\
\hline (010002) & 12 & 1339.51 & 0.556 \\
\hline$(000004)$ & 15 & 1392.32 & 0.537 \\
\hline (001001) & 9 & 1296.06 & 0.515 \\
\hline$(000200)$ & 6 & 1230.04 & 0.474 \\
\hline (000013) & 30 & 1567.80 & 0.456 \\
\hline (010100) & 6 & 1258.37 & 0.413 \\
\hline (100001) & 3 & 1122.63 & 0.400 \\
\hline (010011) & 18 & 1514.99 & 0.354 \\
\hline (000103) & 30 & 1659.26 & 0.292 \\
\hline$(000201)$ & 18 & 1578.12 & 0.260 \\
\hline (000022) & 36 & 1743.28 & 0.233 \\
\hline (010101) & 18 & 1606.45 & 0.227 \\
\hline (000112) & 54 & 1834.74 & 0.223 \\
\hline (001010) & 9 & 1471.54 & 0.219 \\
\hline
\end{tabular}
The states are sorted in the order of decreasing probability. States occupied to $<1 \%$ share $15 \%$ of the total fractional population. The vector $\vec{v}$ denotes the sixuplet $\left(v_{1} v_{2} v_{3} v_{4} v_{5} v_{6}\right)$.

Table II gathers the values of the various partition functions of $\mathrm{SF}_{6}$ at room temperature. The values of the entries suggest that $v_{1}$ is, thermodynamically, the least active mode, for it is both non-degenerated and energetic. The opposite holds true for modes $v_{6}$ and $v_{5}$, and to a lesser extent for mode $v_{4}$, for they are all triply degenerated and of lower energy.

TABLE II. Partition functions of the different normal vibration modes. $n_{i}$ stands for the oscillator dimension. The total vibrational partition function amounts to $Z=3.141$ at $T=295 \mathrm{~K} . v_{i}$ are given in $\mathrm{cm}^{-1}$. The calculation of $Z_{i}$ 's was done analytically.

\begin{tabular}{lcccccc}
\hline \hline Mode & $i=1$ & $i=2$ & $i=3$ & $i=4$ & $i=5$ & $i=6$ \\
\hline$v_{i}$ & 774.55 & 643.35 & 947.98 & 615.02 & 523.56 & 348.08 \\
$n_{i}$ & 1 & 2 & 3 & 3 & 3 & 3 \\
$g_{n_{i}, i}\left(v_{i}\right)$ & 1 & $v_{i}+1$ & & $\frac{1}{2}\left(v_{i}+1\right)\left(v_{i}+2\right)$ & \\
$\left(Z_{i}\right)^{n_{i}}$ & 1.023 & 1.093 & 1.030 & 1.166 & 1.275 & 1.835 \\
\hline \hline
\end{tabular}




\section{Raman cross-sections and hot bands}

\section{First overtones of triply degenerated vibrations}

In the case of a threefold degenerated mode $v_{i}$, the initial state of the molecule is designated by a triplet $\left(v_{i, 1}, v_{i, 2}, v_{i, 3}\right)$ of quantum numbers $v_{i, 1}, v_{i, 2}$, and $v_{i, 3}$, which obey $v_{i}=v_{i, 1}+v_{i, 2}+v_{i, 3}$ and are associated with three equivalent, independent degrees of freedom. As a result, there are six possible final states involved in the upward overtone transition $v_{i} \rightarrow v_{i}+2$, which read: $\left(v_{i, 1}+2, v_{i, 2}, v_{i, 3}\right),\left(v_{i, 1}, v_{i, 2}\right.$ $\left.+2, v_{i, 3}\right),\left(v_{i, 1}, v_{i, 2}, v_{i, 3}+2\right),\left(v_{i, 1}+1, v_{i, 2}+1, v_{i, 3}\right), \quad\left(v_{i, 1}\right.$ $\left.+1, v_{i, 2}, v_{i, 3}+1\right),\left(v_{i, 1}, v_{i, 2}+1, v_{i, 3}+1\right)$. For the first three, the corresponding matrix-elements of the dipole-dipole polarizability tensor invariant $\alpha$ read:

$$
\frac{\sqrt{\left(v_{i, p}+1\right)\left(v_{i, p}+2\right)}}{2}\left(\frac{1}{2 !} \frac{\partial^{2} \alpha}{\partial q_{i, p}^{2}}\right),
$$

where $p=1,2,3$. For the remaining three final states, the matrix-elements are

$$
\frac{\sqrt{\left(v_{i, p}+1\right)\left(v_{i, q}+1\right)}}{2}\left(\frac{1}{2 !} \frac{\partial^{2} \alpha}{\partial q_{i, p} \partial q_{i, q}}+\frac{1}{2 !} \frac{\partial^{2} \alpha}{\partial q_{i, q} \partial q_{i, p}}\right),
$$

with $p, q=1,2,3$ and $p<q$. The derivatives are calculated at the equilibrium position.

Given that the scattering process is incoherent, the interference terms make no net contribution to the intensity, and the Raman zeroth-order moment is simply the sum of the probability averages. Use of symmetry properties further simplifies the calculation of the moment, which reads:

$$
\begin{aligned}
M_{0}= & \frac{3}{16}\left(\frac{\partial^{2} \alpha}{\partial q_{i, 1}^{2}}\right)^{2} \frac{\sum_{v=0}^{\infty} e^{-v x_{i}}(v+1)(v+2)}{\sum_{v=0}^{\infty} e^{-v x_{i}}} \\
& +\frac{3}{4}\left(\frac{\partial^{2} \alpha}{\partial q_{i, 1} \partial q_{i, 2}}\right)^{2}\left(\frac{\sum_{v=0}^{\infty} e^{-v x_{i}}(v+1)}{\sum_{v=0}^{\infty} e^{-v x_{i}}}\right)^{2} .
\end{aligned}
$$

Repeating use $(k=0,1,2)$ of the very useful formula

$$
\sum_{v=0}^{\infty} v^{k} e^{-v x_{i}}=\left(-\frac{d}{d x_{i}}\right)^{k}\left(1-e^{-x_{i}}\right)^{-1}
$$

allows us to calculate the two sum-ratio terms in the rhs of the above equation, which acquire the simple expressions $2\left(1-e^{-x_{i}}\right)^{-2}$ and $\left(1-e^{-x_{i}}\right)^{-2}$, respectively. Thus $M_{0}$ reads:

$$
M_{0}(T)=\frac{3}{8} \gamma_{i}(T)\left[\left(\frac{\partial^{2} \alpha}{\partial q_{i, 1}^{2}}\right)^{2}+2\left(\frac{\partial^{2} \alpha}{\partial q_{i, 1} \partial q_{i, 2}}\right)^{2}\right],
$$

with

$$
\gamma_{i}(T)=\left(1-e^{-\frac{h v_{i}}{k_{B} T}}\right)^{-2}
$$

The quantity $\gamma_{i}(T)$, hereinafter referred to also as the $\gamma$ factor, has the meaning of a "hotband factor," for it describes the scattering cross-section of the overtone at the temperature of the experiment normalized to the cross-section of the same overtone at $0 \mathrm{~K}$.
It was gratifying to find out that the expression of Eq. (7) leads directly to the cross-section for the $\perp$ component $d \sigma_{\perp} / d \Omega$ long ago reported in the analysis of the $2 \nu_{4}$ overtone of cyanogen. ${ }^{53}$ For the latter analysis, a technically exhaustive lengthy mathematical approach had been applied, involving symmetry-adapted normal coordinates and sphericaloscillator algebra mostly addressed to theorists.

\section{The $2 v_{5}$ overtone}

There is sparse evidence of Raman overtones from greenhouse molecules, and only recently has their treatment $\left(\mathrm{CO}_{2}\right.$, $2 v_{3}$ ) begun to be completed. ${ }^{54-57}$ No calibrated spectrum and far too synoptic material on the Raman $2 v_{5}$ overtone of $\mathrm{SF}_{6}$ was found in the so far existing literature, even though evidence for a strongly polarized band has twice been reported. ${ }^{29,30}$ In spite of the very low resolution $\left[6 \mathrm{~cm}^{-1}\right.$ (Ref. 29), 3-10 $\mathrm{cm}^{-1}$ (Ref. 30)] that these scientists have employed in their experiments (far lower than our $0.85 \mathrm{~cm}^{-1}$ ), no or little mention of the role of the hot bands has been made, while the fact of keeping fixed the incident-beam polarization (perpendicular to the scattering plane) further hindered them from reporting on the isotropic spectrum of the overtone.

Given the strongly polarized character of that band, the Raman spectrum of $2 v_{5}$ should merely be isotropic and only derivatives of the mean-polarizability invariant, $\bar{\alpha}$, will be involved in the analysis. Further simplification comes from the observation that the contribution of the cross second derivatives of $\bar{\alpha}$ is zero. A simple argument to show this property is based on symmetry considerations and runs as follows: if we rotate the molecule around $q_{i, 3}$ so that the coordinate $q_{i, 1^{\prime}}$ coincides with $q_{i, 2}$ and the $q_{i, 2^{\prime}}$ switches to $-q_{i, 1}$, the second cross derivative of the polarizability trace changes sign, a result only compatible with $\frac{\partial^{2} \bar{\alpha}}{\partial q_{i, 1} \partial q_{i, 2}}=0$ (endomorphism).

These properties lead to the following expression for the zeroth-order isotropic moment:

$$
M_{0}=\frac{3}{2}\left(1-e^{-\frac{h c v_{i}}{k_{B} T}}\right)^{-2}\left(\frac{1}{2} \frac{\partial^{2} \bar{\alpha}}{\partial q_{5}^{2}}\right)_{\mathrm{eff}}^{2} .
$$

Here, the specification "eff" makes reference to the fact that the second derivative of Eq. (9) has accounted effectively for anharmonic corrections. The second index in $q_{5,1}$ was dropped for simplicity.

The expression of Eq. (9) is formally very different from what would be observed in the absence of hot bands: in their absence, it is the spectral moment $M_{0}^{2 \leftarrow 0}=\lim _{T \rightarrow 0} M_{0}(T)$ of the bare overtone transition $(000020) \leftarrow(000000)$ that would have instead been measured. In the case of room-temperature $\mathrm{SF}_{6}$, one has $P_{\overrightarrow{0}}=0.32$ (see Table I), a value far from being close to 1 . As a result, only ideally can the quantity $M_{0}^{2 \leftarrow 0}$ be isolated from its environment and be measured, especially upon raising gas pressure up to several atmospheres to make the tiny overtone signal be detectable. Interestingly, the low frequency of the $v_{5}$ mode (see Table II) makes the values of $\gamma_{i}(T)$ to literally take off above $T \approx 300 \mathrm{~K}$ and the intensity of the overtone to be doubled at $300{ }^{\circ} \mathrm{C}$ (see below). ${ }^{58}$ 
TABLE III. Energy distribution of fractional $\mathrm{SF}_{6}$ populations and contributions to the hotband $\gamma$-factor of the overtone. For the purpose of this table, these contributions were computed numerically in order to monitor their convergence to the value given by Eq. (8).

\begin{tabular}{lcccc}
\hline \hline Energy $\left(\mathrm{cm}^{-1}\right)$ & $<1000$ & $<2000$ & $<3000$ & $<4000$ \\
Number of states & 15 & 157 & 852 & 3183 \\
Fractional population $(\%)$ & 80.71 & 97.88 & 99.86 & 99.99 \\
Contribution to the $\gamma$-factor & 0.884 & 1.137 & 1.172 & 1.175 \\
\hline \hline
\end{tabular}

\section{Distribution of the scattered intensity over energy}

According to our computations, at room temperature, 98\% of the contributions to the gas population and to the hotband $\gamma$-factor of Eq. (8) are from vibrational states with energies lying below $2000 \mathrm{~cm}^{-1}$ and $v_{5} \leq 2$. This is inferred from the data of Tables III and IV. The first of these tables allows one to analyze these contributions as a function of the width of the energy window and of the number of vibrational states considered in the computation. The second table displays how the population of the gas molecules and their contributions to the $2 v_{5}$ Raman signal are shared on the $v_{5}$ ladder.

Figure 1 illustrates the way in which the molecular population of $\mathrm{SF}_{6}$ (boxes) and its contribution to the $\gamma$-factor of Eq. (8) (lines) are distributed for the $2 v_{5}$ overtone, as a function of vibrational energy at room temperature. On climbing the energy ladder, the convergence to the result of Eq. (8) for $2 v_{5}$ is enough fast to make mechanical anharmonicity not to have a significant effect at room temperature. This convergence is considered effective as soon as the energy has reached $2100 \mathrm{~cm}^{-1}$.

Figures 2 and 3 show how molecular population of $\mathrm{SF}_{6}$ (dark grey sticks) along with contributions to the hotband $\gamma$ factor of Eq. (8) (light grey sticks) are distributed as a function of $v_{t o t}\left(=\sum_{i=1}^{6} v_{i}\right)$ and $v_{5}$, respectively. As seen from these figures, in order for the convergence to the $\gamma_{i}(295 \mathrm{~K})$ value to be almost complete, it suffices to climb the $v$-steps up to $v_{t o t}=5$ and $v_{5}=2$. This finding suggests that the anharmonicity of the $\mathrm{SF}_{6}$ potential should have only a reduced effect in the treatment of $2 v_{5}$ at room temperature.

Figure 4 illustrates the behavior of the hotband factor of Eq. (8) as a function of temperature (in K), for three representative $2 v_{i}$ overtones of $\mathrm{SF}_{6}$ (solid line curves). These are in the descending order: $2 v_{6}$ (black solid line), $2 v_{5}$ (blue solid line), $2 v_{1}$ (red solid line). Note that the most significant variations are due to the low energy of the corresponding modes

TABLE IV. Fractional populations of $\mathrm{SF}_{6}$ molecules and their contributions to the Raman intensity of the overtone for different values of the initial state vibrational number $v_{5}$ at $295 \mathrm{~K}$. All contributions due to other modes have already been accounted for in the calculation of the entry values.

\begin{tabular}{lcc}
\hline \hline$v_{5}$ & $P\left(v_{5}\right)(\%)$ & Scattered intensity $(\%)$ \\
\hline 0 & 78.56 & 66.88 \\
1 & 18.22 & 25.85 \\
2 & 2.82 & 6.00 \\
3 & 0.36 & 1.08 \\
4 & 0.04 & 0.17 \\
5 & 0.00 & 0.02 \\
\hline \hline
\end{tabular}

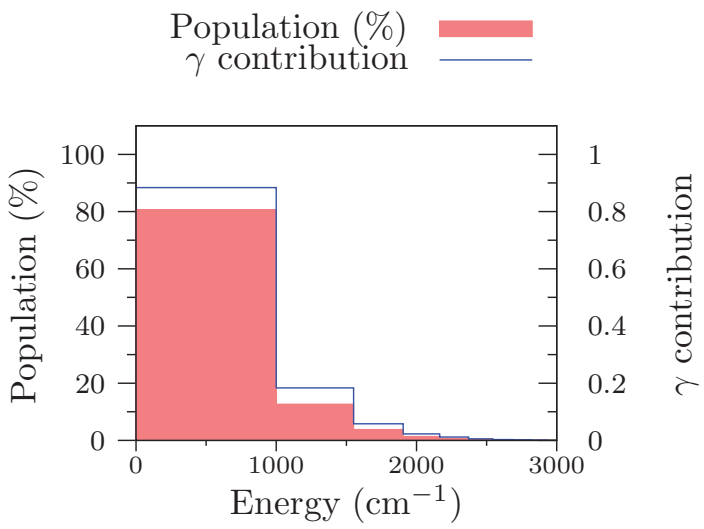

FIG. 1. Fractional population of gas molecules (boxes) and contributions to the overtone $\gamma$-factor of Eq. (8) (lines) as a function of vibrational energy range (in units of $\mathrm{cm}^{-1}$ ) at $295 \mathrm{~K}$.

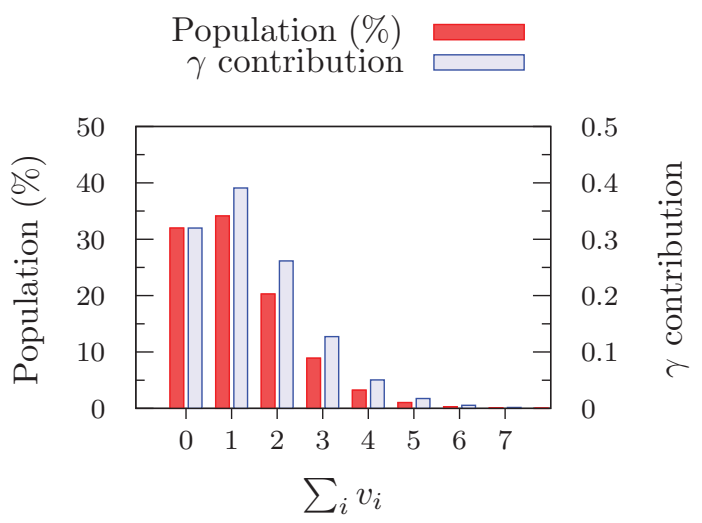

FIG. 2. Fractional population of gas molecules (dark grey sticks) and contributions to the overtone $\gamma$-factor of Eq. (8) (light grey sticks) as a function of $v_{t o t}=\sum_{i} v_{i}$ at $295 \mathrm{~K}$.

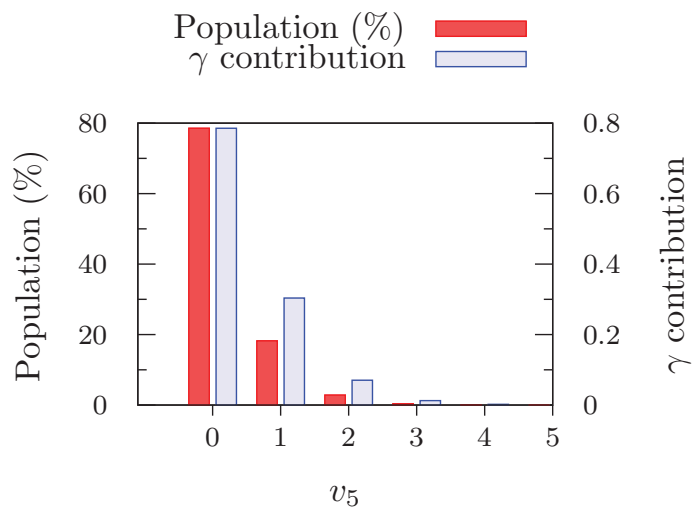

FIG. 3. Fractional population of gas molecules (dark grey sticks) and contributions to the overtone $\gamma$-factor of Eq. (8) (light grey sticks) as a function of $v_{5}$ at $295 \mathrm{~K}$. 


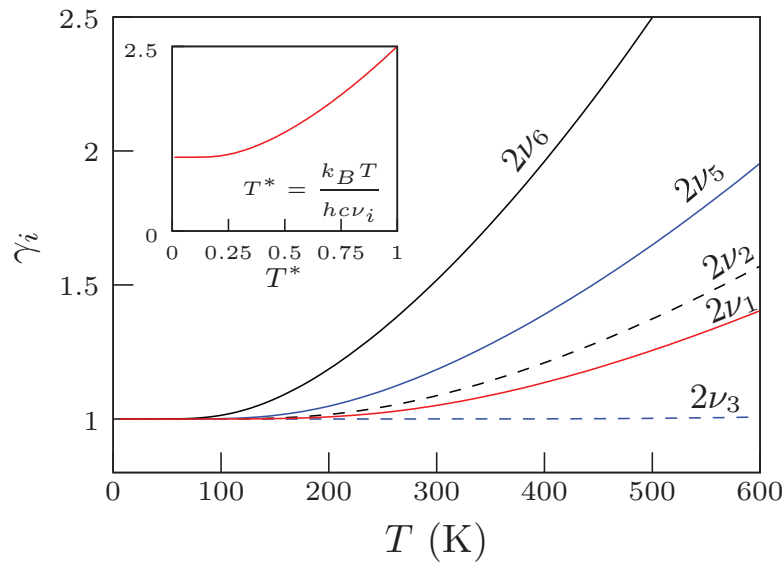

FIG. 4. Temperature dependence of the $\gamma$-factor for three overtones of $\mathrm{SF}_{6}$ (solid line curves). From top to bottom: $2 v_{6}$ (black solid line), $2 v_{5}$ (blue solid line), $2 v_{1}$ (red solid line). For comparison, the factors of the $2 v_{2}$ and $2 v_{3}$ overtones of $\mathrm{CO}_{2}$ have also been plotted (dashed line curves). In the inset, the $\gamma$-factor is shown as a function of reduced temperature $T^{*}=k_{B} T /\left(h c v_{i}\right)$.

(348 and $524 \mathrm{~cm}^{-1}$ for $v_{6}$ and $v_{5}$, respectively). The more energetic $v_{1}$ mode $\left(774 \mathrm{~cm}^{-1}\right)$ is by far less affected by temperature. For the sake of comparison, the factors that are relative to the $2 v_{3}$ and $2 v_{2}$ overtones of $\mathrm{CO}_{2}$ are also shown (dashed line curves). To make it possible to compare, whatever the molecule, how the hotband factor depends simultaneously on $T$ and $v_{i}$, a reduced temperature $T^{*}=\frac{k_{B} T}{h c v_{i}}$ was introduced ${ }^{59}$ and the plot of $\gamma_{i}$ as a function of $T^{*}$ is shown in the inset. The value $T^{*}=1$ corresponds to a fictitious mode with energy equal to that of the thermal excitation; at room temperature, this mode is activated at $205 \mathrm{~cm}^{-1}$. Below $T^{*}=0.2$, the hotband factor makes almost no effect. At room temperature, this behavior would correspond to modes with frequencies greater than $1025 \mathrm{~cm}^{-1}$. None of the $\mathrm{SF}_{6}$ modes has such a high frequency. In contrast, the two stretching modes of $\mathrm{CO}_{2}$ do belong to this class, and the impact of the thermal factor for $\mathrm{CO}_{2}$ becomes tiny.

\section{EXPERIMENT}

The experiment was carried out using equipment well known for its sensitivity and a meticulous and stiff protocol that we have established to ensure reliability in the signal extraction. Description in detail of the equipment and of the way we are used to operate has been given previously. ${ }^{13,14,17,60,61}$ Technical details, related to the way in which the $2 v_{5}$ signal was isolated from its environment and to how a densityindependent absolute-calibrated isotropic spectrum was extracted from the recorded spectrum, will be skipped. In what follows, only the essentials of the isotropic spectrum are gathered.

\section{A. Setup, settings, and detection}

A solid-state 532-nm frequency-doubled Nd:YV04 laser was used to shine green light onto high purity (99.995\%) pressurized sulfur hexafluoride gas. The sample was maintained at a constant temperature of $294.5 \pm 1 \mathrm{~K}$ and confined in a four- window cell specially designed to support high pressures. Raman signals scattered thereby at a right angle were recorded. A nitrogen-cooled CCD was used for the detection. The laser power was kept constant at $2 \mathrm{~W}$ for all the runs. Various gas densities covering the range from 2 to 27 amagat were used, that is, pressures going from $\sim 2$ to $20 \mathrm{~atm}$.

The center of the $2 v_{5}$ overtone was seen at $1048 \mathrm{~cm}^{-1}$ and the band was recorded over a wide frequency interval, $v$, ranging from 980 to $1150 \mathrm{~cm}^{-1}$. At each experimental run and setting value for the gas density, calibration of the Raman signal (photons/second) was made to an absolute-scale densitydependent (amagat $\mathrm{cm}^{3}$ ) signal intensity $S(v)$ by means of the $\mathrm{S}_{0}(1)$ rotational line of molecular hydrogen. This line was centered at $587.1 \mathrm{~cm}^{-1}$. A switchable linear polarization for the incident beam was used, giving rise to independent Raman signals $S_{\|}(v)$ and $S_{\perp}(v)$. These signals were then combined together in a linear combination with appropriate coefficients ${ }^{55}$ to form the isotropic signal $S_{i s o}(v)$. To make sure that the recorded spectrum was indeed due to transitions by single $\mathrm{SF}_{6}$ molecules, a rigorous protocol was applied, enabling us to determine the exact way in which the gas density, $\rho$, affects the integrated signal $\left(S_{i s o}\right)_{\text {int }}$. A behavior that is strictly linear in $\rho$ was observed, which is compelling evidence that the isotropic spectrum was indeed due to isolated $\mathrm{SF}_{6}$ molecules. The density-independent isotropic intensity profile was then deduced through $I_{i s o}(v)=S_{i s o}(v) / \rho$.

In Figure 5, the absolute-calibrated density-dependent spectra $S_{i s o}$ are illustrated on a semi logarithmic scale as a function of Raman frequency (in $\mathrm{cm}^{-1}$ ), for the various gas densities employed and after extraction of the surrounding parasitic signals. The deleted stripe seen on the plot around the frequency $1034.7 \mathrm{~cm}^{-1}$ corresponds to the position where the hydrogen rotational line $\mathrm{S}_{0}(3)$ appeared and perturbed the $\mathrm{SF}_{6}$ spectrum. This range was covered by interpolation before any integration of spectra. The straight line shown in the

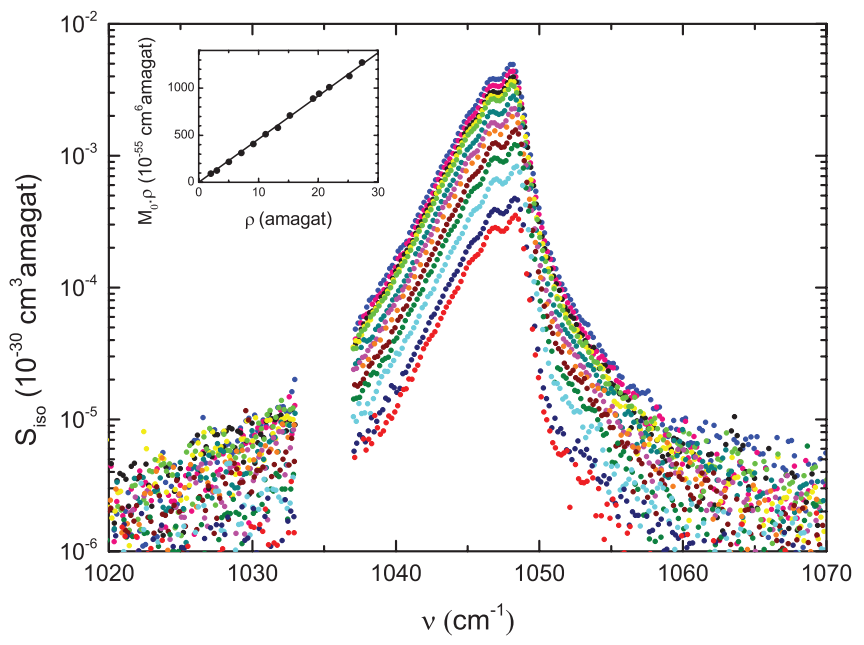

FIG. 5. Absolute-calibrated spectra $S_{\text {iso }}$ (in units of $\mathrm{cm}^{3}$ amagat) as a function of Raman frequency $v$ (in units of $\mathrm{cm}^{-1}$ ) for 13 values of gas density ranging from 2 to 27 amagat (in the upward direction). In the inset, the product $M_{0} \cdot \rho\left[M_{0}\right.$ is the experimental zeroth-order moment (in units of $\mathrm{cm}^{6}$ ) of the signal generated from a gas sample of density $\rho$ ] is shown as a function of $\rho$ (in units of amagat). The linear dependence is evidence that the recorded band comes from isolated molecules alone. 


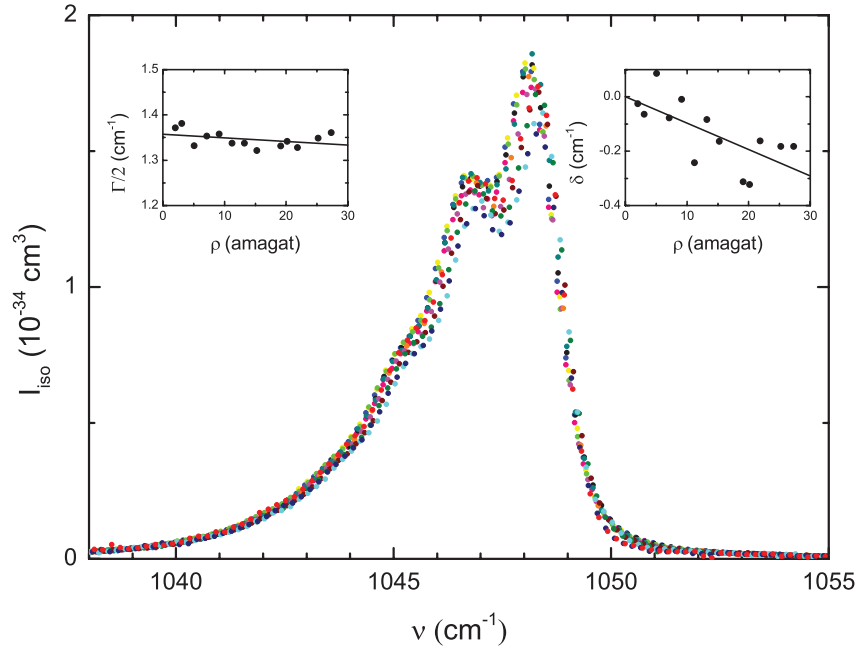

FIG. 6. Isotropic spectrum $I_{\text {iso }}$ (in units of $\mathrm{cm}^{3}$ ) as a function of Raman frequency $v$ (in units of $\mathrm{cm}^{-1}$ ) for 13 values of gas density ranging from 2 to 27 amagat (in the upward direction; same colors as in Fig. 5). The small differences between the spectra (especially at the top) is an indication of weak but distinguishable pressure-induced effects modifying the shape of the overtone. In the insets the half-width $\Gamma / 2$ at $1 / \sqrt{e}$-maximum and the band-top shift $\delta$ (in units of $\mathrm{cm}^{-1}$ ) are shown as a function of density $\rho$ (in units of amagat).

inset represents the quantity $M_{0} \rho$ as a function of $\rho$, with $M_{0}$ the zeroth-order isotropic moment. Given the definition of $M_{0}$ [see below Eq. (10) ], the perfect alignment of the points in the inset is evidence for spectrum integrals $\int_{-\infty}^{\infty} S_{i s o}(\rho, v) d v$ scaling linearly with $\rho$.

Figure 6 shows the isotropic spectrum, $I_{i s o}$, as a function of $v$, for the different values of $\rho$. The close resemblance of the spectra is indication of small yet observable pressureinduced effects for this overtone. The precise way in which broadening and shift were found to depend on $\rho$ is shown in the insets: A tiny negative broadening (narrowing), scaling linearly with $\rho$ and amounting to $-8 \times 10^{-4} \mathrm{~cm}^{-1} /$ amagat is seen along with a redshift about ten times greater. The small dispersion of points, both in the main body of the figure and in the insets, is a measure of the quality of our measurements.

\section{B. Accounting for the surrounding bands}

A major difficulty in the study of the overtone came from the far wing of certain transitions such as the very intense fundamental $v_{1}$ (centered at $774 \mathrm{~cm}^{-1}$ ) or the collision-induced component of $v_{3}$ (centered at $948 \mathrm{~cm}^{-1}$ ), which severely perturbed the baseline of the $2 v_{5}$ signal. Another perturbation occurred due to the presence of undesired signals located at the upper frequency side of the $2 v_{5}$ overtone, which were interpreted as being possibly due to the far wings of the $v_{2}$ $+v_{5}$ combination band and to a lesser extent due to the $2 v_{4}$ and $2 v_{2}$ overtones, centered at 1166,1230 , and $1285 \mathrm{~cm}^{-1}$, respectively. The perturbation of the latter three signals, although substantial, was less significant than that of the lower frequency signal. For all of them, a systematic least-squares fitting procedure was applied employing an exponential wing profile (adapted to the perfectly linear aspect seen on a logarithmic plot for the spectral tails) and 13 experimental runs for
TABLE V. Scattering cross-sections for the overtone by using incident light polarized $\perp$ to the scattering plane.

$\begin{array}{ll}\frac{d \sigma_{\perp}}{d \Omega}\left(\times 10^{-33} \mathrm{~cm}^{2} \mathrm{sr}^{-1}\right) & \\ \text { This work } & 0.96 \\ \text { Previous works } & 0.93^{\mathrm{a}} \\ & 0.61^{\mathrm{b}}\end{array}$

${ }^{a}$ Reference 30

${ }^{\mathrm{b}}$ Reference 29.

gas densities covering the range from 2.03 to 27.34 amagat. Thorough analysis of that careful procedure will be reported elsewhere. The spectra of Figures 5 and 6 were obtained after extraction of the surrounding parasitic signals.

\section{The spectral moment}

The isotropic zeroth-order moment was calculated by integrating the isotropic spectral intensity $I_{i s o}\left(=\frac{S_{i s o}}{\rho}\right)$, by means of the formula:

$$
M_{0}=\left[2 \pi\left(v_{0}-v_{s}\right)\right]^{-4} \int_{-\infty}^{+\infty} I_{i s o}(v) d v .
$$

In the latter expression, $v_{0}$ denotes the laser wavenumber and $v_{s}$ the wavenumber at the center of the recorded band. The result is in units of $\mathrm{cm}^{6}$. We obtained the value $M_{0}$ (expt.) $=4.59(69) \times 10^{-54} \mathrm{~cm}^{6}$.

Table $\mathrm{V}$ gathers previous and present results for scattering cross-sections obtained with incident beam polarization perpendicular to the scattering plane.

\section{CALCULATIONS}

\section{A. Derivatives}

Given the value of $M_{0}$ extracted from the recorded isotropic spectrum, we are now able to deduce, on the basis of Eq. (9), the value $\left|\left(\partial^{2} \bar{\alpha} / \partial q_{5}^{2}\right)_{\text {eff }}\right|=0.0218 a_{0}^{3}\left(a_{0}\right.$ is the Bohr radius). Further refinement of the analysis is possible upon using anharmonicity data that are either available or that are yet to come. Thus, by assuming that the anharmonicity corrections are independent of the vibration quantum number (which is a reasonable assumption for enough low-lying vibrational excitations $v_{5}$ and $v_{\text {tot }}$; see Table IV, Figures 2 and $3)$, the harmonic derivative $\partial^{2} \bar{\alpha} / \partial q_{5}^{2}$ reads:

$$
\frac{\partial^{2} \bar{\alpha}}{\partial q_{5}^{2}}=\left(\frac{\partial^{2} \bar{\alpha}}{\partial q_{5}^{2}}\right)_{\mathrm{eff}}-\frac{\partial \bar{\alpha}}{\partial q_{1}} k_{155} \frac{2 v_{1}}{4 v_{5}^{2}-v_{1}^{2}} .
$$

This expression stems from the most general expression ${ }^{62}$ relevant to overtone transition matrix-elements $\left\langle\cdots v_{i}+2 \cdots|\bar{\alpha}| \cdots v_{i} \cdots\right\rangle$ :

$$
\begin{aligned}
\left(\frac{\partial^{2} \bar{\alpha}}{\partial q_{i}^{2}}\right)_{\text {eff }}= & \frac{\partial^{2} \bar{\alpha}}{\partial q_{i}^{2}}+\frac{\partial \bar{\alpha}}{\partial q_{i}} \frac{2 k_{i i i}}{v_{i}} \\
& +\sum_{k \neq i}\left[\frac{\partial \bar{\alpha}}{\partial q_{k}} k_{k i i} \frac{2 v_{k}}{4 v_{i}^{2}-v_{k}^{2}}\right]
\end{aligned}
$$

upon setting $i=5, k=1$, and $\partial \bar{\alpha} / \partial q_{i}=0$. The latter condition is a consequence of the fact that $v_{5}$ is a vibration that is 
TABLE VI. Values for $\left(\partial^{2} \bar{\alpha} / \partial q_{i}^{2}\right)_{\text {eff }}$ and $\left(\partial^{2} \bar{\alpha} / \partial q_{i}^{2}\right)$ in units of $a_{0}^{3}$.

\begin{tabular}{lrrrr}
\hline \hline$\left(\frac{\partial^{2} \bar{\alpha}}{\partial q_{5}^{2}}\right)_{\text {eff }}$ & & $\frac{\partial^{2} \bar{\alpha}}{\partial q_{5}^{2}}$ & & \\
+0.0218 & $0.020^{\mathrm{a}, \mathrm{b}}$ & $0.020^{\mathrm{a}, \mathrm{c}}$ & $0.074^{\mathrm{b}, \mathrm{d}}$ & $0.072^{\mathrm{c}, \mathrm{d}}$ \\
-0.0218 & $-0.024^{\mathrm{a}, \mathrm{b}}$ & $-0.024^{\mathrm{a}, \mathrm{c}}$ & $0.030^{\mathrm{b}, \mathrm{d}}$ & $0.028^{\mathrm{c}, \mathrm{d}}$ \\
\hline \hline
\end{tabular}

a The value $k_{155}=0.68 \mathrm{~cm}^{-1}$ was used (taken from Ref. 45).

${ }^{\mathrm{b}}$ The value $\partial \bar{\alpha} / \partial q_{1}=0.975 a_{0}^{3}$ was used (taken from Ref. 63, expt.).

${ }^{\mathrm{c}}$ The value $\partial \bar{\alpha} / \partial q_{1}=0.937 a_{0}^{3}$ was used (taken from Ref. 19, calculations).

${ }^{\mathrm{d}}$ The value $k_{155}=-17 \mathrm{~cm}^{-1}$ was used (taken from Ref. 44).

not totally symmetric and, as such, its fundamental transition gives rise merely to a depolarized spectrum. So too is the case with all the other derivatives $\partial \bar{\alpha} / \partial q_{k}$ in the summation over $k$, except for $k=1$ : of the six vibrations of $\mathrm{SF}_{6}, v_{1}$ is the only one to be totally symmetric $\left(\mathrm{A}_{1}\right)$.

The sign uncertainty in the value of the first rhs term in the above equation along with the very different magnitudes that have been reported for the cubic force constant $k_{155}$ (Refs. 44 and 45) gives rise to a multitude of values for $\partial^{2} \bar{\alpha} / \partial q_{5}^{2}$. All of them were compatible with our spectrum. They are gathered in Table VI. The value of $\partial \bar{\alpha} / \partial q_{1}$ is known to a good accuracy both experimentally ${ }^{63}$ and numerically (on the basis of second-order Møller-Plesset perturbation theory) ${ }^{19}$ but given the poor accuracy of $k_{155}$, the $\partial^{2} \bar{\alpha} / \partial q_{5}^{2}$ values are given to three decimal places.

Table VII gathers all the input data that were needed to apply Eq. (11). Polarizability derivatives are given in $a_{0}^{3}$; vibrational frequencies for the two modes and their cubic force constant are in $\mathrm{cm}^{-1}$.

\section{B. Higher order overtones}

Before closing this section, it is worth noting that Eq. (9) is restricted to first overtones. It was almost effortless to extend it further to make it include overtones of any order, such as second overtones $\left(\Delta v_{i}=3\right)$ which are IR-allowed and known for their challenging spectra. A remarkably elegant formula is then obtained for the $\gamma$-factor along with a closed-form expression for the integrated isotropic spectral moment in the case of overtone bands " $\left(\Delta v_{i}\right) v_{i}$ " of arbitrary order for a non-degenerated vibration:

$$
\begin{aligned}
\gamma_{i}^{\left(\Delta v_{i}\right)} & =\left(1-e^{-\frac{h c v_{i}}{k_{B} T}}\right)^{-\Delta v_{i}} \\
M_{0} & =\frac{1}{2^{\Delta v_{i}}\left(\Delta v_{i}\right) !} \gamma_{i}^{\left(\Delta v_{i}\right)}\left(\frac{\partial^{\Delta v_{i}} \bar{\alpha}}{\partial q_{i, x}^{\Delta v_{i}}}\right)^{2} .
\end{aligned}
$$

TABLE VII. Input data needed in Eq. (11). Polarizability derivatives are in $a_{0}^{3}$ units; vibrational frequencies and force constants are in $\mathrm{cm}^{-1}$.

\begin{tabular}{lccc}
\hline \hline$\nu_{1}$ & $\nu_{5}$ & $\frac{\partial \bar{\alpha}}{\partial q_{1}}$ & $k_{155}$ \\
774.55 & 523.56 & $0.975^{\mathrm{a}}$ & $0.68^{\mathrm{b}}$ \\
& & $0.937^{\mathrm{c}}$ & $-17^{\mathrm{d}}$ \\
\hline \hline
\end{tabular}

${ }^{a}$ Expt.: Ref. 63.

${ }^{\mathrm{b}}$ A b initio calculations: Ref. 45 .

${ }^{\mathrm{c}}$ Ab initio calculations: Ref. 19.

${ }^{\mathrm{d}}$ Ab initio calculations: Ref. 44.
Particular interesting is the fact that the hotband effect is greatly enhanced with increasing overtone order. For certain vibrations, the intensity of the $3 v_{i}$ band can even be doubled at room temperature.

Note that, in spite of the diverging behavior $\lim _{h \rightarrow 0} \gamma_{i}^{\left(\Delta v_{i}\right)}=\infty$, the moments given above remain finite at the classical limit $(h \rightarrow 0)$ :

$$
M_{0}^{\text {class }}=\frac{1}{2^{\Delta v_{i}}\left(\Delta v_{i}\right) !}\left(\frac{k_{B} T}{\mu_{i}\left(2 \pi c v_{i}\right)^{2}}\right)^{\Delta v_{i}}\left(\frac{\partial^{\Delta v_{i}} \bar{\alpha}}{\partial r_{i, x}^{\Delta v_{i}}}\right)^{2},
$$

$r_{i}\left[=\sqrt{\hbar /\left(2 \pi c v_{i} \mu_{i}\right)} q_{i}\right]$ and $\mu_{i}$ being the physical length coordinate and the reduced mass ${ }^{64}$ associated with $q_{i}$, respectively, and $\partial^{\Delta v_{i}} \bar{\alpha} / \partial r_{i, x}^{\Delta v_{i}}$ the corresponding $\Delta v_{i}$-order derivative. Equation (14) shows that integrated intensities go with $v_{i}$ as $\left(T / v_{i}^{2}\right)^{\Delta v_{i}}$. Interestingly enough, changes in $v_{i}$ have a more pronounced effect on integrated spectra than they have it on bare $\gamma$-factors $\left[\gamma_{i}^{\left(\Delta v_{i}\right)} \sim\left(T / v_{i}\right)^{\Delta v_{i}}\right.$, see Figure 4 for $\left.\Delta v_{i}=2\right]$.

\section{SYNOPSIS}

Highly accurate absolute-calibrated Raman spectra were recorded from room-temperature pressurized $\mathrm{SF}_{6}$ around the frequency of the highly polarized first overtone of the scissoring vibration $v_{5}\left(1048 \mathrm{~cm}^{-1}\right)$. Experiments with 13 gas densities, $\rho$, ranging between 2 and 27 amagat were run. The profiles were seen to be only little affected by pressure and to obey rigorously a linear law in $\rho$. A systematic protocol was applied to remove effects due to the spectral wings of the accidentally present surrounding Raman bands, allowing us to rigorously determine the isotropic overtone spectrum. Full account was made of the multitude of hot bands around 1048 $\mathrm{cm}^{-1}$, and a formula for $\partial^{2} \bar{\alpha} / \partial q_{5}^{2}$ was worked out involving a $T$-independent integrated intensity $M_{0}(T) / \gamma(T)$. Also, the issue of the much challenging IR-allowed second overtones was addressed in this article, through generalization of our formulas to elegant expressions that are applicable to overtones of any order for non-degenerated modes. In view of the growing attention that the issues of radiative forcing are being receiving internationally and of the fast $8 \%$ annual increase of $\mathrm{SF}_{6}$ gas in the air, there is urgent need for ab initio calculated values of dipole moment and polarizability second derivatives like $\partial^{2} \bar{\alpha} / \partial q_{5}^{2}$. Not much seems to have emerged in that field since Maroulis's contributions. ${ }^{19}$ Not only a fuller comparison between theory and experiment will in such a way be possible, also a reliable value for the thus-far loosely determined cubic force constant $k_{155}$ will at last become available.

\section{ACKNOWLEDGMENTS}

One of us (D.K.) gratefully acknowledges doctoral fellowship support from the CNRS and "la Région des Pays de la Loire."

${ }^{1}$ G. C. Tabisz, in Molecular Spectroscopy (a Specialist Periodical Report), edited by R. F. Barrow, D. A. Long and J. Sheridan, (Chemical Society, London, 1979), Vol. 6, pp. 136-173.

${ }^{2}$ E. Bright Wilson Jr., J. C. Decius, and Paul C. Cross, Molecular Vibrations: The Theory of Infrared and Raman Vibrational Spectra (Dover, New York, 1980). 
${ }^{3}$ D. A. Long, The Raman Effect: A Unified Treatment of the Theory of Raman Scattering by Molecules (Wiley, Chichester, UK, 2002).

${ }^{4}$ L. Frommhold, Adv. Chem. Phys. 46, 1 (1981).

${ }^{5}$ Collision- and Interaction-Induced Spectroscopy, edited by G. C. Tabisz and M. N. Neuman (Kluwer, Dordrecht, 1995).

${ }^{6}$ T. Bancewicz, Y. Le Duff, and J.-L. Godet, Adv. Chem. Phys. 119, 267 (2001).

${ }^{7}$ L. Frommhold, Collision-Induced Absorption in Gases (Cambridge University Press, New York, 2006).

${ }^{8}$ J.-M. Hartmann, C. Boulet, and D. Robert, Collisional Effects on Molecular Spectra: Laboratory Experiments and Model, Consequences for Applications (Elsevier, Amsterdam, 2008).

${ }^{9}$ G. Avila, G. Tejeda, J. M. Fernandez, S. Montero, J. Mol. Spectrosc. 220, 259 (2003).

${ }^{10}$ J.-M. Hartmann, J.-P. Bouanich, K. W. Jucks, Gh. Blanquet, J. Walrand, D. Bermejo, J.-L. Domenech, and N. Lacome, J. Chem. Phys. 110, 1959 (1999).

${ }^{11}$ V. Boudon and N. Lacome, J. Mol. Spectrosc. 222, 291 (2003).

${ }^{12}$ V. Boudon, J. L. Domenech, D. Bermejo, and H. Willner, J. Mol. Spectrosc. 228, 392 (2004).

${ }^{13}$ F. Rachet, M. Chrysos, C. Guillot-Noël, and Y. Le Duff, Phys. Rev. Lett. 84, 2120 (2000).

${ }^{14}$ F. Rachet, Y. Le Duff, C. Guillot-Noël, and M. Chrysos, Phys. Rev. A 61, 062501 (2000).

${ }^{15}$ Y. Le Duff, Phys. Rev. Lett. 90, 193001 (2003).

${ }^{16}$ M. Chrysos, A. P. Kouzov, N. I. Egorova, and F. Rachet, Phys. Rev. Lett. 100, 133007 (2008).

${ }^{17}$ S. Dixneuf, M. Chrysos, and F. Rachet, J. Chem. Phys. 131, 074304 (2009).

${ }^{18}$ S. M. El-Sheikh, G. C. Tabisz, and R. T. Pack, J. Chem. Phys. 92, 4234 (1990).

${ }^{19}$ G. Maroulis, Chem. Phys. Lett. 312, 255 (1999).

${ }^{20} \mathrm{~T}$. Bancewicz, J.-L. Godet, and G. Maroulis, J. Chem. Phys. 115, 8547 (2001).

${ }^{21}$ J.-L. Godet, F. Rachet, Y. Le Duff, K. Nowicka, and T. Bancewicz, J. Chem. Phys. 116, 5337 (2002).

${ }^{22}$ Y. Le Duff, J.-L. Godet, T. Bancewicz, and K. Nowicka, J. Chem. Phys. 118, 11009 (2003).

${ }^{23}$ I. A. Verzhbitskiy, M. Chrysos, F. Rachet, and A. P. Kouzov, Phys. Rev. A 81, 012702 (2010)

${ }^{24}$ M. Chrysos and I. A. Verzhbitskiy, Phys. Rev. A 81, 042705 (2010).

${ }^{25}$ I. A. Verzhbitskiy, M. Chrysos, and A. P. Kouzov, Phys. Rev. A 82, 052701 (2010).

${ }^{26}$ J.-P. Champion, M. Loëte, and G. Pierre, "Spherical top spectra," in Spectroscopy of the Earth's Atmosphere and Interstellar Medium, edited by K. N. Rao and A. Weber Editors (Academic Press, New York, 1992), pp. 339-422.

${ }^{27}$ A. R. Ravishankara, S. Solomon, A. A. Turnipseed, and R. F. Warren, Science 259, 194 (1993).

${ }^{28}$ M. K. W. Ko, N. D. Sze, W.-C. Wang, G. Shia, A. Goldman, F. J. Murcray, D. G. Murcray, and C. P. Rinsland, J. Geophys. Res. 98, 10499, doi:10.1029/93JD00228 (1993).

${ }^{29}$ W. Holzer and R. Ouillon, Chem. Phys. Lett. 24, 589 (1974).

${ }^{30}$ D. P. Shelton and L. Ulivi, J. Chem. Phys. 89, 149 (1988).

${ }^{31}$ S. Montero, J. Chem. Phys. 79, 4091 (1983).

${ }^{32}$ W. C. Marlow, Proc. Phys. Soc. 86, 731 (1965).

${ }^{33}$ W. Kolos and L. Wolniewicz, J. Chem. Phys. 46, 1426 (1967).

${ }^{34}$ Y. Y. Milenko, L. V. Karnatsevich, and V. S. Kogan, Physica 60, 90 (1972).

${ }^{35}$ A. S. Pine and A. G. Robiette, J. Mol. Spectrosc. 80, 388 (1980).

${ }^{36}$ D. P. Hodgkinson and A. J. Taylor, Mol. Phys. 52, 1017 (1984).

${ }^{37}$ H. B. Levene and D. S. Perry, J. Chem. Phys. 80, 1772 (1984).

${ }^{38}$ R. Harzer, G. Schweizer, and K. Selter, J. Mol. Spectrosc. 132, 310 (1988).

${ }^{39}$ D. McNaughton and C. Evans, J. Mol. Spectrosc. 182, 342 (1997).

${ }^{40}$ V. Boudon, M. Hepp, M. Herman, I. Pak, and G. Pierre, J. Mol. Spectrosc. 192, 359 (1998).

${ }^{41}$ U. Hohm and K. Kerl, Mol. Phys. 58, 541 (1986).

${ }^{42}$ U. Hohm and K. Kerl, Mol. Phys. 61, 1295 (1987).
${ }^{43}$ W. B. Clodius and C. R. Quade, J. Chem. Phys. 82, 2365 (1985).

${ }^{44}$ B. J. Krohn and J. Overend, J. Phys. Chem. 88, 564 (1984).

${ }^{45}$ D. P. Hodgkinson, J. C. Barrett, and A. G. Robiette, Mol. Phys. 54, 927 (1985).

${ }^{46}$ J.-Q. Chen, F. Iachello, and J.-L. Ping, J. Chem. Phys. 104, 815 (1996).

${ }^{47}$ This is to be contrasted with the large values taken by constants related to stretching of the S-F bond, such as $k_{133}$, and with the agreement between theory [ $-55 \mathrm{~cm}^{-1}$ (Ref. 44), $-56.75 \mathrm{~cm}^{-1}$ (Ref. 45)], and experiment $\left[-56.25 \mathrm{~cm}^{-1}\right.$ (Ref. 45)] that has been found for $k_{133}$.

${ }^{48}$ P. P. Bera, J. S. Francisco, and T. J. Lee, J. Phys. Chem. A 113, 12694 (2009). According to these scientists "Molecules possessing several F atoms will always have a large radiative forcing parameter in the calculation of their global warming potential." This is a consequence of the "linear increase in the bond dipole derivatives for the molecule, which leads to a quadratic increase in infrared (IR) intensity."

${ }^{49}$ The reader should be mindful that it is the first excited state of each mode that specifies the mode's degeneracy $\left[n_{i}=g_{n_{i}, i}(1)\right]$. Note also that for the ground vibrational level of each mode, the expression of Eq. (3) gives $g_{n_{i}, i}(0)=1$.

${ }^{50}$ R. S. McDowell, B. J. Krohn, H. Flicker, and M. C. Vasquez, Spectrochim. Acta A 42, 351 (1986).

${ }^{51}$ G. Herzberg, Molecular Spectra and Molecular Structure II: Infrared and Raman Spectra of Polyatomic Molecules (Van Nostrand Company, Toronto, 1966), Eq. (V,17).

${ }^{52}$ It is more practical and informative, in terms of interpretation, to use a definition for the vibrational partition function in which the zero-point energy is absent. In this way $\left(Z_{i}\right)^{-n_{i}}$ becomes a probability [the probability $P_{i}\left(v_{i}=0\right)$ that the ground-state of mode $i$ is occupied] and $\left(Z_{i}\right)^{n_{i}}$ takes values greater than and close to 1 . Parenthetically, this choice is consistent with the definition $Z_{\text {elec }}=g_{\text {elec }} \exp \left(D_{0} / k_{B} T\right)$ for the electronic partition function of $\mathrm{SF}_{6}$, where $g_{\text {elec }}$ is the electronic degeneracy and $D_{0}=D_{e}-\sum_{i} \frac{1}{2} n_{i} h c v_{i}$ the binding energy of the molecule, the latter energy being expressed as a difference between the equilibrium energy $D_{e}$ and the zero-point energy $\sum_{i} \frac{1}{2} n_{i} h c v_{i}$ : by making this subtraction the zeropoint energy has been implicitly incorporated into $Z_{\text {elec }}$ and has thus been "removed" from the vibrational partition function.

${ }^{53}$ S. Montero, J. Chem. Phys. 72, 2347 (1980).

${ }^{54}$ M. Chrysos, I. A. Verzhbitskiy, F. Rachet, and A. P. Kouzov, J. Chem. Phys. 134, 044318 (2011).

${ }^{55}$ M. Chrysos, I. A. Verzhbitskiy, F. Rachet, and A. P. Kouzov, J. Chem. Phys. 134, 104310 (2011).

${ }^{56}$ I. A. Verzhbitskiy, A. P. Kouzov, F. Rachet, and M. Chrysos, J. Chem. Phys. 134, 194305 (2011).

${ }^{57}$ I. A. Verzhbitskiy, A. P. Kouzov, F. Rachet, and M. Chrysos, J. Chem. Phys. 134, 224301 (2011).

${ }^{58}$ This behavior is to be contrasted with the one met in the recently treated ${ }^{54-57} 2 v_{3} \mathrm{CO}_{2}$ overtone. There, the $2 v_{3}$ overtone had been identified as the combined interaction " $\left(00^{0} 2\right) \leftarrow\left(00^{0} 0\right)$ and $\left(01^{1} 2\right) \leftarrow\left(01^{1} 0\right)$," found to include at $295 \mathrm{~K}$ the whole amount of the scattered intensity to proportions $92.8 \%$ and $7.2 \%$, respectively. ${ }^{56}$ Although, formally, some thermal factor should too have been included in those studies, the concept had not yet been revealed to us: owing to the high frequency of the $\mathrm{CO}_{2} \nu_{3}$ mode $\left(2349 \mathrm{~cm}^{-1}\right), \gamma_{3}(295 \mathrm{~K})$ amounts to 1.00002 .

${ }^{59}$ The meaning of the "reduced temperature" becomes clearer after the definition $T^{*}=T / T_{v i b}$, where $T_{v i b}=h c v_{i} / k_{B}$ is the "vibrational temperature."

${ }^{60}$ S. Dixneuf, M. Chrysos, and F. Rachet, Phys. Rev. A 80, 022703 (2009).

${ }^{61}$ M. Chrysos, S. Dixneuf, and F. Rachet, Phys. Rev. A 80, 054701 (2009).

${ }^{62}$ T. D. Kolomiitsova and D. N. Shchepkin, in Advances in Spectroscopy, edited by R. J. H. Clark and R. E. Hester (Wiley, Chichester, 1995), Vol. 23.

${ }^{63}$ D. A. Long and E. L. Thomas, Trans. Faraday Soc. 59, 1026 (1963).

${ }^{64}$ For $q_{1}$, we have $\mu_{1}=6 m_{F}$, with $m_{F}$ the atomic mass of fluorine [see for instance L. G. Gerchikov and G. F. Gribakin, Phys. Rev. A 77, 042724 (2008); see also Ref. 23; for $\mathrm{CF}_{4}$, see Ref. 6, p. 299]. The formula $q_{1}$ $=\sqrt{\mu_{1} \omega_{1} / \hbar} \Delta R$ makes the link between the dimensionless normal stretch coordinate $q_{1}$ and the elongation $\Delta R$ of the S-F bond. 